\title{
New and emerging zoophilic dermatophytes in Europe
}

\author{
Cmokova, $A^{1,2}-$ Guillot, $J^{3}-$ Hoyer, $L^{4}-$ Kano, $R^{5}-$ Nenoff, $P^{6}-$ Peano, $A^{7}-$ Risco-Castillo, V 8 \\ Cabañes, FJ9 - Stubbe, D ${ }^{10}-$ Uhrlaß, $S^{5}-$ Hubka, V1,2
}

${ }^{1}$ Faculty of Science, Charles University, Prague, Czech Republic, ${ }^{2}$ Institute of Microbiology of the CAS, Prague, Czech Republic; ${ }^{3}$ Service de Parasitologie-Mycologie Ecole Nationale Vétérinaire d'AlfortMaisons Alfort Cedex France; ${ }^{8}$ Department of Pathobiology, University of Illinois Urbana-Champaign, Urbana, USA; 5 Nihon University School of Veterinary Medicine, Fujisawa, Japan; ${ }^{6}$ Laboratory for Medical Microbiology Mölbis, Germany; ${ }^{7}$ Facoltà di Medicina Veterinaria, Università degli Studi di Torino, Turin, Italy; ${ }^{8}$ Unit of Parasitology, Mycology, Parasite and Fungal Diseases, Paris, France, ${ }^{9}$ Department de Patologia i Producció Animals, Universitat Autònoma de Barcelona Bellaterra, Barcelona, España; ${ }^{10}$ BCCM/IHEM, Biomedical fungi and yeasts collection, Scientific Institute of Public Health, Brussels, Belgium

Trichophyton benhamiae, T. erinacei and related species are increasingly reported as a cause of dermatophytosis in pet or companion animals and their breeders or owners worldwide. A considerable genetic and phenotypic variability has been revealed in these emerging pathogens. To substantiate the initial finding, we assembled strains isolated from various hosts in different European countries, Africa, North America, and Japan. We conducted DNA sequencing of several genetic loci, microsatellite analysis, analysis of morphology, and physiological testing to elucidate whether the detected level of variability reflects undescribed species diversity or a high infraspecific variability.

Only three poorly defined populations were found among $T$. erinacei isolates. These populations were strongly host-specific. First (E1) and second (E2) population were specific for African hedgehogs (Atelerix albiventris); population E1 for wild animals in Africa, while populazion E2 for pet animals in Europe. Consequently, population E2 is also responsible for the majority of mycoses in pet owners $(89 \%$ of human isolates examined). The last population of $T$. erinacei (E3) was isolated exclusively from European hedgehogs.

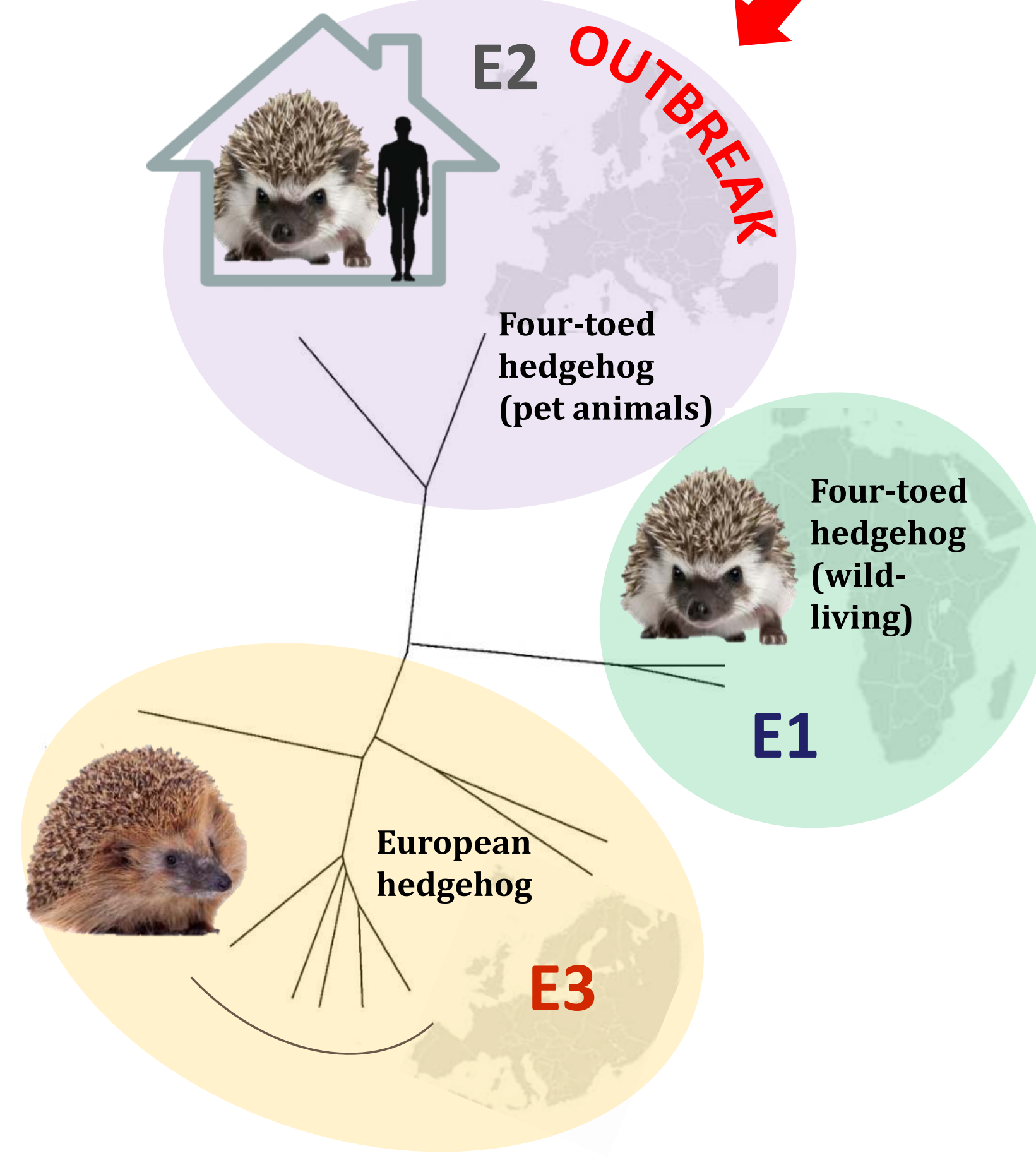

A dendrogram showing population structure of T. ERINACEI. The tree is based on eight microsatellite markers characterized in 146 strains, and was constructed using UPGMA algoritm.

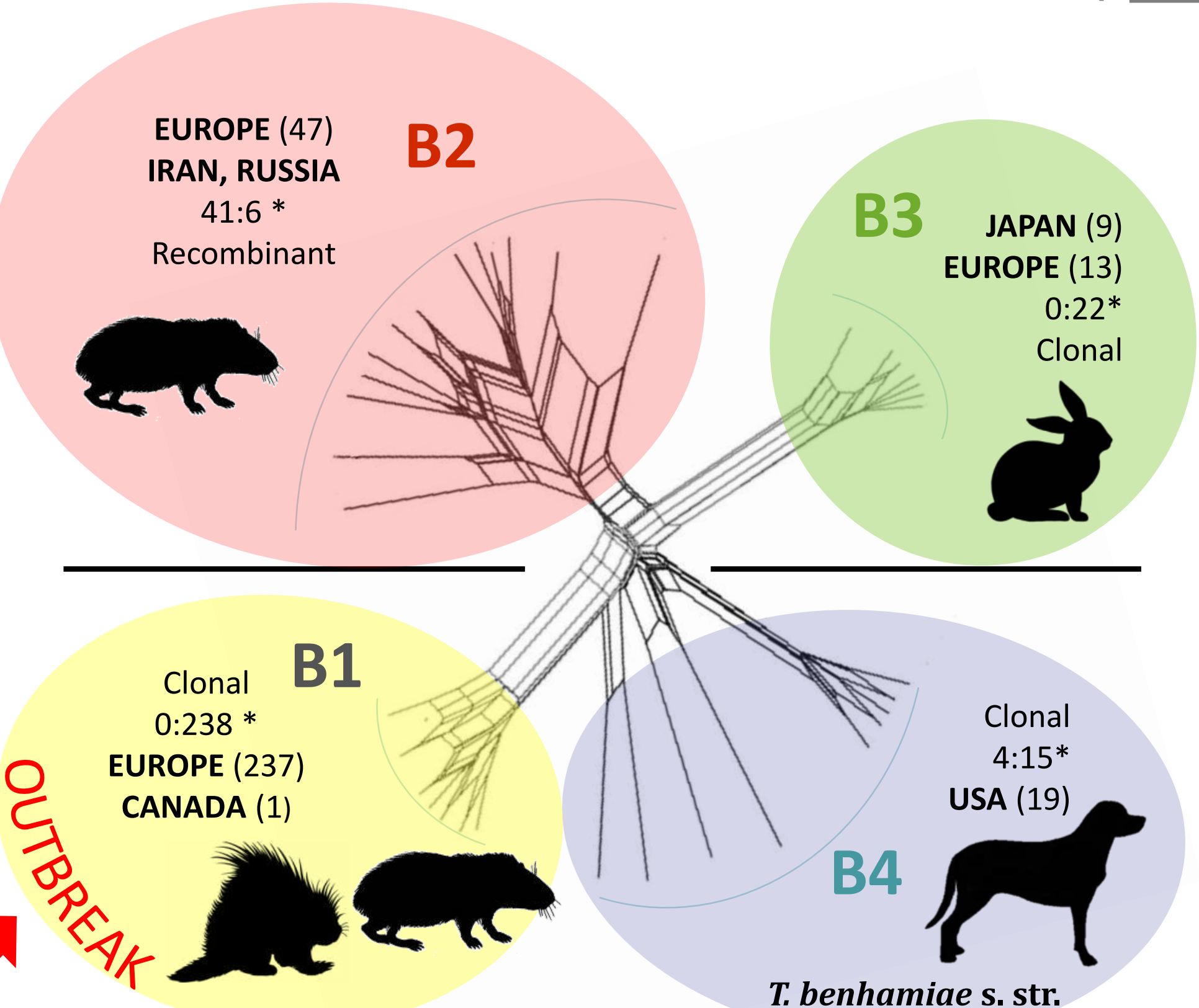

A neighbour-net network showing population structure of $T$. BENHAMIAE. The network is based on ten microsatellite markers characterized in 326 strains of human and animal origin.

The project was made possible thanks to grants GAUK 600217 grant of the Czech Ministry of Health (AZV 17-31269A). Vit Hubka is grateful for support from Czechoslovak Microscopy Society (CSMS scholarship 2016).
Among $326 T$. benhamiae isolates, four distinct populations correcponding to species were revealed, including three undescribed species (B1-B3). Species B1 and B2 are abundantly reported from Europe and have guinea pig as an exclusive host there. In addition, species B1 was reported from North American Porcupine in Canada. These species differ strikingly by phenotype, physiology, produced secondary metabolites and reproductive strategy (sexual versus clonal). Species B1 is responsible for the current epidemic of dermatophytosis in Europe. Trichophyton benhamiae s. str. (B4) occurs mostly in America and is associated with infections in dogs, while species B3 has been isolates in Europe and Japan from rabbits and their owners. We suggest that the most virulent species B1 was introduced to Europe from North America where the species B1 was found in North American porcupine. In addition, North America is the center of genetic diversity of $T$. benhamiae and native population/species B4 ( $T$. benhamiae s. str.) is closely related to clonal species B1 that was absent in Europe untill 2004. 